\title{
CONTINUITY OF THE POLAR DECOMPOSITION FOR UNBOUNDED OPERATORS ON HILBERT C*-MODULES
}

\author{
KAMRAN SHARIFI \\ Shahrood University of Technology, Iran
}

\begin{abstract}
For unbounded operators $t, s$ between Hilbert $\mathrm{C}^{*}$-modules which admit the polar decompositions $\mathcal{V}|t|, \mathcal{W}|s|$, respectively, we obtain an explicit upper bound estimate for the gap between $t$ and $s$ in terms of the norm of the bounded operators $\mathcal{V}-\mathcal{W}, \mathcal{C}_{|t|}-\mathcal{C}_{|s|}$ and $\mathcal{C}_{\left|t^{*}\right|}-\mathcal{C}_{\left|s^{*}\right|}$, where $\mathcal{C}_{|t|}$ and $\mathcal{C}_{|s|}$ are the Cayley transforms of $|t|$ and $|s|$. The result are used to drive a criterion for continuity of the polar decomposition for unbounded operators between Hilbert $\mathrm{C}^{*}$-modules.
\end{abstract}

\section{INTRODUCTION}

A Hilbert $\mathrm{C}^{*}$-module is an object like a Hilbert space except that the inner product is not scalar-valued, but takes its values in a $\mathrm{C}^{*}$-algebra of coefficients. Some fundamental properties of Hilbert spaces like Pythagoras' equality, self-duality, and even decomposition into orthogonal complements must be given up. They play an important role in the modern theory of $\mathrm{C}^{*}$-algebras and the study of locally compact quantum groups.

A (left) pre-Hilbert $C^{*}$-module over an arbitrary $\mathrm{C}^{*}$-algebra $\mathcal{A}$ is a left $\mathcal{A}$-module $E$ equipped with an $\mathcal{A}$-valued inner product $\langle\cdot, \cdot\rangle: E \times E \rightarrow \mathcal{A}$, which is $\mathcal{A}$-linear in the first variable and has the properties:

$\langle x, y\rangle=\langle y, x\rangle^{*}, \quad\langle x, x\rangle \geq 0$ with equality if and only if $x=0$.

A pre-Hilbert $\mathcal{A}$-module $E$ is called a Hilbert $\mathcal{A}$-module if $E$ is a Banach space with respect to the norm $\|x\|=\|\langle x, x\rangle\|^{1 / 2}$. A Hilbert $\mathcal{A}$-submodule $E$ of a Hilbert $\mathcal{A}$-module $F$ is orthogonally complemented if $E$ and its orthogonal complement $E^{\perp}:=\{y \in F:\langle x, y\rangle=0$ for all $x \in E\}$ yield $F=E \oplus E^{\perp}$. For

2010 Mathematics Subject Classification. 46L08, 46C50, 47C15, 47B50.

Key words and phrases. Hilbert $\mathrm{C}^{*}$-module, unbounded operator, gap metric, Cayley transform. 
the basic theory of Hilbert $\mathrm{C}^{*}$-modules we refer to the book by E. C. Lance [10] and the papers $[4,11]$.

Throughout the present paper we assume $\mathcal{A}$ to be an arbitrary $\mathrm{C}^{*}$-algebra (not necessarily unital). We deal with bounded and unbounded operators at the same time, so we denote bounded operators by capital letters and unbounded operators by small letters. We use the notations $\operatorname{Dom}(),. \operatorname{Ker}($. and $\operatorname{Ran}($.$) for domain, kernel and range of operators, respectively.$

We denote by $B(E, F)$ the set of all adjointable operators from a Hilbert $\mathcal{A}$-module $E$ to another Hilbert $\mathcal{A}$-module $F$, i.e., of all maps $T: E \rightarrow F$ such that there exists $T^{*}: F \rightarrow E$ with the property $\langle T x, y\rangle=\left\langle x, T^{*} y\right\rangle$ for all $x \in E, y \in F$. $B(E, E)$ is abbreviated by $B(E)$.

The gap topology is induced by the metric $d(t, s)=\left\|P_{G(t)}-P_{G(s)}\right\|$ where $P_{G(t)}$ and $P_{G(s)}$ are projections onto the graphs of densely defined closed operators $t, s$, respectively. The gap topology has been studied systematically in the book [8] and the papers $[3,9,12,14]$ and references therein.

An unbounded regular operator between Hilbert $\mathrm{C}^{*}$-modules is an analogue of a closed operator on a Hilbert space. A closed and densely defined operator $t$ from a Hilbert $\mathrm{C}^{*}$-module $E$ to another Hilbert $\mathrm{C}^{*}$-module $F$ is called regular if its adjoint $t^{*}$ is also densely defined and if the range of $\left(1+t^{*} t\right)$ is dense in $E$. The author and M. Frank in [6] have given necessary and sufficient conditions for unbounded regular operators to admit polar decomposition. Suppose $\mathcal{V}|t|$ and $\mathcal{W}|s|$ are the polar decompositions of unbounded regular operators $t$ and $s$, and $d$ denotes the gap distance between $t$ and $s$. In the present paper we give an explicit upper bound for $d(t, s)$ in terms of the quantities $\left\|\mathcal{C}_{|t|}-\mathcal{C}_{|s|}\right\|,\left\|\mathcal{C}_{\left|t^{*}\right|}-\mathcal{C}_{\left|s^{*}\right|}\right\|$ and $\|\mathcal{V}-\mathcal{W}\|$, in which $\mathcal{C}_{|t|}, \mathcal{C}_{|s|}$ are the Cayley transforms of selfadjoint regular operators $|t|,|s|$, respectively. This result enables us to find a criterion for continuity of the polar decomposition for unbounded regular operators. The author believes that the final results, i.e., Theorem 3.2 and Corollary 3.3 can be regarded as the new results even in the case of Hilbert spaces.

\section{Preliminaries}

Unbounded regular operators were first introduced by Baaj and Julg in [2], later they were studied more by Woronowicz in [16], while investigating noncompact quantum groups. Suppose $E, F$ are Hilbert $\mathcal{A}$-modules, an $\mathcal{A}$ linear operator $t: \operatorname{Dom}(t) \subseteq E \rightarrow F$ is said to be regular if

(i) $t$ is closed and densely defined with domain $\operatorname{Dom}(t)$,

(ii) its adjoint $t^{*}$ is also densely defined, and

(iii) the range of $1+t^{*} t$ is dense in $E$.

Note that if we set $\mathcal{A}=\mathbb{C}$, i.e., if we take $E, F$ to be Hilbert spaces, then this is exactly the definition of a densely defined closed operator, except that in that case, both the second and the third condition follow from the first 
one. We denote the set of all regular operators from $E$ to $F$ by $R(E, F)$. In case $E=F, R(E, E)$ is denoted by $R(E)$. If $t$ is regular then $t^{*}$ and $t^{*} t$ are regular, and $t=t^{* *}$. Define $Q_{t}:=\left(1+t^{*} t\right)^{-1 / 2}, R_{t}:=\left(1+t^{*} t\right)^{-1}=Q_{t}^{2}$ and $F_{t}:=t\left(1+t^{*} t\right)^{-1 / 2}=t Q_{t}$, then $\operatorname{Ran}\left(Q_{t}\right)=\operatorname{Dom}(t), 0 \leq Q_{t} \leq 1$ in $B(E)$ and $F_{t} \in B(E, F)([10,(10.4)])$. The bounded operator $F_{t}$ is called the bounded transform of the regular operator $t$. The map $t \rightarrow F_{t}$ defines a bijection

$R(E, F) \rightarrow\left\{T \in B(E, F):\|T\| \leq 1\right.$ and $\operatorname{Ran}\left(1-T^{*} T\right)$ is dense in $\left.F\right\}$.

This map is adjoint-preserving, i.e., $F_{t}^{*}=F_{t^{*}}$, cf. [10, Theorem 10.4]. In particular, we have $\left\|F_{t}\right\| \leq 1$ and $t R_{t}=F_{t} Q_{t} \in B(E, F)$ which imply $\left\|t R_{t}\right\|=$ $\left\|F_{t} Q_{t}\right\| \leq\left\|F_{t}\right\|\left\|Q_{t}\right\| \leq 1$.

Corollary 2.1. Suppose $t: \operatorname{Dom}(t) \subseteq E \rightarrow F$ is an unbounded regular operator, then $F_{t} F_{t}^{*}=1-R_{t}^{*}$ and $\left(t R_{t}\right)^{*}=t^{*} R_{t^{*}}$

Proof. The first equality follows from the fact that $Q_{t^{*}}=\left(1-F_{t} F_{t^{*}}\right)^{1 / 2}$. Since $F_{t}^{*}=F_{t^{*}}$, we have $\left(t Q_{t}\right)^{*}=t^{*} Q_{t^{*}}$. Applying [6, Remark 2.2] to the regular operator $t^{*}$, we obtain $t^{*} Q_{t^{*}}^{2}=Q_{t} t^{*} Q_{t^{*}}$. We therefore have $\left(t R_{t}\right)^{*}=$ $\left(t Q_{t} Q_{t}\right)^{*}=Q_{t}^{*}\left(t Q_{t}\right)^{*}=Q_{t} t^{*} Q_{t^{*}}=t^{*} Q_{t^{*}}^{2}=t^{*} R_{t^{*}}$.

Suppose $t \in R(E, F)$, the absolute value of $t$ is defined by $|t|:=\left(t^{*} t\right)^{1 / 2}$, then $|t|$ is a positive regular operator and satisfies $\operatorname{Dom}(|t|)=\operatorname{Dom}(t)$. Basic definitions and a few simple facts about regular operators on Hilbert $\mathrm{C}^{*}$ modules can be found in [10, chapters 9 and 10], and the papers $[5,16]$ with details.

Let $t \in R(E, F)$ then $E \oplus F=G(t) \oplus V\left(G\left(t^{*}\right)\right)$, where $V \in B(E \oplus F, F \oplus$ $E)$ is defined by $V(x, y)=(y,-x)$, cf. [10, Theorem 9.3]. The orthogonal projection $P_{G(t)}: E \oplus F \rightarrow E \oplus F$ can be described through the following matrix

$$
P_{G(t)}=\left(\begin{array}{cc}
R_{t} & t^{*} R_{t^{*}} \\
t R_{t} & 1-R_{t^{*}}
\end{array}\right) \in B(E \oplus F) .
$$

To see this, just use $[10,(9.7)]$ and Corollary 2.1.

Definition 2.2. Let $t, s \in R(E, F)$ then the gap metric on the space of all unbounded regular operators is defined by $d(t, s)=\left\|P_{G(t)}-P_{G(s)}\right\|$ where $P_{G(t)}$ and $P_{G(s)}$ are orthogonal projections onto $G(t)$ and $G(s)$, respectively. The topology induced by this metric is called gap topology.

Let $E, F$ be two Hilbert $\mathcal{A}$-modules and operators $t, s$ be in $R(E, F)$. An equivalent picture of the gap metric is now definable by using (2.1) as well as the fact that $\left(t R_{t}\right)^{*}=t^{*} R_{t^{*}}$. Indeed, the following metric, which is again denoted by $d$, is uniformly equivalent to the gap metric

$$
d(t, s):=\sup \left\{\left\|R_{t}-R_{s}\right\|,\left\|R_{t^{*}}-R_{s^{*}}\right\|,\left\|t R_{t}-s R_{s}\right\|\right\} .
$$

This fact follows from (2.1) and [14, Remark 1.1]. The metric (2.2) shows that the gap metric is stable under taking adjoint operation, i.e., $d(t, s)=d\left(t^{*}, s^{*}\right)$. 
Proposition 2.3. Suppose $d$ is the gap metric and $T, S$ are bounded adjointable operators in $B(E, F)$. Then

$$
\begin{aligned}
& {\left[\left(1+\|T\|^{2}\right)(1+\|S\|)\right]^{-1}\|T-S\|} \\
& \quad \leq d(T, S) \leq \max \{\|T\|+\|S\|, 1+\|S\|(\|S\|+\|T\|)\}\|T-S\| .
\end{aligned}
$$

In particular, the metric which is given by the usual norm of bounded operator and the gap metric $d$ are equivalent on the space of all bounded adjointable operators.

Proof. In view of the equality $R_{T}-R_{S}=R_{T}\left(S^{*} S-T^{*} T\right) R_{S}$ and the facts that $\left\|R_{T}\right\| \leq 1$ and $\left\|R_{S}\right\| \leq 1$, we have

$$
\begin{aligned}
\left\|R_{T}-R_{S}\right\| & \leq\left\|R_{T}\right\|\left\|S^{*} S-T^{*} T\right\|\left\|R_{S}\right\| \\
& \leq\left\|S^{*}(S-T)+\left(S^{*}-T^{*}\right) T\right\| \\
& \leq\left\|S^{*}\right\|\|S-T\|+\left\|S^{*}-T^{*}\right\|\|T\| \\
& =(\|T\|+\|S\|)\|T-S\|, \\
\left\|T R_{T}-S R_{S}\right\| & =\left\|(T-S) R_{T}+S\left(R_{T}-R_{S}\right)\right\| \\
& \leq\|T-S\|+\|S\|\left\|R_{T}-R_{S}\right\| \\
& \leq\|T-S\|(1+\|S\|(\|S\|+\|T\|)) .
\end{aligned}
$$

Similarly $\left\|R_{T^{*}}-R_{S^{*}}\right\| \leq(\|T\|+\|S\|)\|T-S\|$. The above inequalities and the expression (2.2) imply that $d(T, S) \leq M_{1}\|T-S\|$, where $M_{1}=\max \{\|T\|+$ $\|S\|, 1+\|S\|(\|S\|+\|T\|)\}$. We know that $T-S=(T-S) R_{T} R_{T}^{-1}$ and $(T-S) R_{T}=T R_{T}-S R_{S}+S\left(R_{S}-R_{T}\right)$, so we obtain

$$
\begin{aligned}
\|T-S\| & \leq\left\|(T-S) R_{T}\right\|\left\|R_{T}^{-1}\right\| \\
& \leq\left(1+\|T\|^{2}\right)\left\|(T-S) R_{T}\right\| \\
& \leq\left(1+\|T\|^{2}\right)\left(\left\|T R_{T}-S R_{S}\right\|+\|S\|\left\|R_{S}-R_{T}\right\|\right) \\
& \leq\left(1+\|T\|^{2}\right)(d(T, S)+\|S\| d(T, S)) \\
& \leq\left(1+\|T\|^{2}\right)(1+\|S\|) d(T, S) .
\end{aligned}
$$

Therefore $M_{2}\|T-S\| \leq d(T, S)$, for $M_{2}=\left[\left(1+\|T\|^{2}\right)(1+\|S\|)\right]^{-1}$.

Following [12], the space of all bounded adjointable operators between Hilbert $\mathrm{C}^{*}$-modules is an open dense subset of the space of all unbounded regular operators with respect to the gap topology. As we stated in Proposition 2.3, the restriction of the gap topology on the space of all bounded adjointable operators is equivalent with the topology which is generated by the usual operator norm. However, the uniform structures induced by the gap metric and by the operator norm on the space of bounded adjointable operators are different. This follows from the fact that the metric which is given by the usual norm of bounded operator is complete while the gap metric on the set of bounded adjointable operators is not complete. 
Remark 2.4. Let $E$ be a Hilbert $\mathcal{A}$-module and $S R(E)$ be the set of all selfadjoint elements of $R(E)$. Suppose $t \in S R(E)$, then [10, Lemmata 9.7, 9.8] imply that the operators $t \pm i$ are injective and surjective, in addition

$\mathcal{C}_{t}: S R(E) \longrightarrow \mathcal{C}=\{U \in B(E): U$ is unitary and $1-U$ has dense range $\}$,

$$
t \mapsto \mathcal{C}_{t}=(t-i)(t+i)^{-1}
$$

is a bijection which is called the Cayley transform of $t$, cf. [10, Theorem 10.5]. The Cayley transform $\mathcal{C}_{t}$ can be written as $\mathcal{C}_{t}=1-2 i(t+i)^{-1}$. Thus $(t+i)^{-1}-(s+i)^{-1}=\frac{i}{2}\left(\mathcal{C}_{t}-\mathcal{C}_{s}\right)$, for each $t, s \in S R(E)$.

Proposition 2.5. On the space $S R(E)$ the gap metric is uniformly equivalent to the metric $\tilde{d}$ given by

(2.3) $\tilde{d}(t, s):=\left\|(t+i)^{-1}-(s+i)^{-1}\right\|=\frac{1}{2}\left\|\mathcal{C}_{t}-\mathcal{C}_{s}\right\|$, for all $t, s \in S R(E)$.

Proof. Suppose $t, s$ are selfadjoint regular operators on $E$ then the gap metric can be written as $d(t, s)=\sup \left\{\left\|R_{t}-R_{s}\right\|,\left\|t R_{t}-s R_{s}\right\|\right\}$. The operators $t \pm i$ are bijections and so

$$
\begin{aligned}
& (t-i)^{-1}=(t+i)\left(t^{2}+1\right)^{-1}=t R_{t}+i R_{t}, \\
& (t+i)^{-1}=(t-i)\left(t^{2}+1\right)^{-1}=t R_{t}-i R_{t},
\end{aligned}
$$

which imply

$$
\begin{aligned}
R_{t} & =\frac{1}{2 i}\left((t-i)^{-1}-(t+i)^{-1}\right), \\
t R_{t} & =\frac{1}{2}\left((t-i)^{-1}+(t+i)^{-1}\right) .
\end{aligned}
$$

On the other hand every bounded adjointable operator and its adjoint operator have the same norm, use this fact and the equalities (2.4) and (2.5), we can infer that

$$
\frac{1}{2} \tilde{d}(t, s) \leq d(t, s) \leq \tilde{d}(t, s) .
$$

\section{The POLAR DECOMPOSITION AND CONTINUITY}

We begin this section with the notification that bounded and unbounded module maps between Hilbert $\mathrm{C}^{*}$-modules are generally not decomposable into their polar forms, cf. $[6,15]$. However, the author and M. Frank in [6, Theorem 3.1] gave a necessary and sufficient condition.

Theorem 3.1. Suppose $E, F$ are Hilbert $\mathcal{A}$-modules and $t \in R(E, F)$ denotes a regular operator then the following conditions are equivalent:

(i) $t$ has a unique polar decomposition $t=\mathcal{V}|t|$, where $\mathcal{V} \in B(E, F)$ is a partial isometry for which $\operatorname{Ker}(\mathcal{V})=\operatorname{Ker}(t), \operatorname{Ker}\left(\mathcal{V}^{*}\right)=\operatorname{Ker}\left(t^{*}\right)$, $\operatorname{Ran}(\mathcal{V})=\overline{\operatorname{Ran}(t)}$ and $\operatorname{Ran}\left(\mathcal{V}^{*}\right)=\overline{\operatorname{Ran}(|t|)}=\overline{\operatorname{Ran}\left(t^{*}\right)}$. 
(ii) $E=\operatorname{Ker}(|t|) \oplus \overline{\operatorname{Ran}(|t|)}$ and $F=\operatorname{Ker}\left(t^{*}\right) \oplus \overline{\operatorname{Ran}(t)}$.

In this situation, $\mathcal{V}^{*} \mathcal{V}|t|=|t|, \mathcal{V}^{*} t=|t|$ and $\mathcal{V} \mathcal{V}^{*} t=t$.

The above theorem and [5, Proposition 2.2] show that regular operators with closed range have always polar decomposition. Recall that an operator $\mathcal{V}$ in $B(E, F)$ is a partial isometry if $F_{0}=\operatorname{Ran}(\mathcal{V})$ is complemented in $F$ and there exists a complemented submodule $E_{0}$ of $E$ such that $\mathcal{V}$ is isometric from $E_{0}$ onto $F_{0}$ and $\mathcal{V}\left(E_{0}^{\perp}\right)=0$. Like the general theory of Hilbert spaces, $\mathcal{V}$ is a partial isometry if and only if $\mathcal{V}^{*} \mathcal{V}$ is a projection in $B(E)$, if and only if $\mathcal{V} \mathcal{V}^{*}$ is a projection in $B(F)$, if and only if $\mathcal{V} \mathcal{V}^{*} \mathcal{V}=\mathcal{V}$, if and only if $\mathcal{V}^{*} \mathcal{V} \mathcal{V}^{*}=\mathcal{V}^{*}$ (see e.g. $[10,13])$.

TheOREM 3.2. Let $t, s \in R(E, F)$ have the uniqe polar decompositions $t=\mathcal{V}|t|, s=\mathcal{W}|s|$, respectively. Then

(i) $d(t, s) \leq\|\mathcal{V}-\mathcal{W}\|+\frac{1}{2}\left\|\mathcal{C}_{|t|}-\mathcal{C}_{|s|}\right\|+\frac{1}{2}\left\|\mathcal{C}_{\left|t^{*}\right|}-\mathcal{C}_{\left|s^{*}\right|}\right\|$, and

(ii) $\left\|\mathcal{C}_{|t|}-\mathcal{C}_{|s|}\right\| \leq 4 d(t, s)+4\|\mathcal{V}-\mathcal{W}\|$

Proof. Suppose $\mathcal{V}|t|, \mathcal{W}|s|$ are the polar decomposition of regular operators $t, s$. Since $R_{t}=\left(1+t^{*} t\right)^{-1}=\left(1+|t|^{2}\right)^{-1}=R_{|t|}$ and $R_{s}=R_{|s|}$, we have $\left\|R_{t}-R_{s}\right\|=\left\|R_{|t|}-R_{|s|}\right\| \leq d(|t|,|s|)$. Analogously, $\left\|R_{t^{*}}-R_{s^{*}}\right\|=$ $\| R_{\left|t^{*}\right|}-R_{\left|s^{*}\right|} \mid \leq d\left(\left|t^{*}\right|,\left|s^{*}\right|\right)$. The equality $t R_{t}-s R_{s}=\mathcal{V}\left(|t| R_{|t|}-|s| R_{|s|}\right)+$ $(\mathcal{V}-\mathcal{W})|s| R_{|s|}$ together with the facts that $\|\mathcal{V}\| \leq 1$ and $\left\||s| R_{|s|}\right\| \leq 1$ imply that

$$
\begin{aligned}
\left\|t R_{t}-s R_{s}\right\| & \leq\|\mathcal{V}\|\left\||t| R_{|t|}-|s| R_{|s|}\right\|+\|\mathcal{V}-\mathcal{W}\|\left\||s| R_{|s|}\right\| \\
& \leq\|\mathcal{V}\| d(|t|,|s|)+\|\mathcal{V}-\mathcal{W}\| \\
& \leq d(|t|,|s|)+\|\mathcal{V}-\mathcal{W}\| .
\end{aligned}
$$

In view of (2.3) and (2.6), the preceding inequalities imply

$$
\begin{aligned}
d(t, s) & =\sup \left\{\left\|R_{t}-R_{s}\right\|,\left\|R_{t^{*}}-R_{s^{*}}\right\|,\left\|t R_{t}-s R_{s}\right\|\right\} \\
& \leq\|\mathcal{V}-\mathcal{W}\|+d(|t|,|s|)+d\left(\left|t^{*}\right|,\left|s^{*}\right|\right) \\
& \leq\|\mathcal{V}-\mathcal{W}\|+\frac{1}{2}\left\|\mathcal{C}_{|t|}-\mathcal{C}_{|s|}\right\|+\frac{1}{2}\left\|\mathcal{C}_{\left|t^{*}\right|}-\mathcal{C}_{\left|s^{*}\right|}\right\|
\end{aligned}
$$

This proves (i).

To prove (ii) we note for $\mathcal{V}^{*} t=|t|$ and $\mathcal{W}^{*} s=|s|$ the identities

$$
|t| R_{|t|}-|s| R_{|s|}=\mathcal{V}^{*} t R_{t}-\mathcal{W}^{*} s R_{s}=\mathcal{V}^{*}\left(t R_{t}-s R_{s}\right)+\left(\mathcal{V}^{*}-\mathcal{W}^{*}\right) s R_{s} .
$$

Consequently,

$$
\begin{aligned}
\left\||t| R_{|t|}-|s| R_{|s|}\right\| & \leq\left\|\mathcal{V}^{*}\right\|\left\|t R_{t}-s R_{s}\right\|+\left\|\mathcal{V}^{*}-\mathcal{W}^{*}\right\|\left\|s R_{s}\right\| \\
& \leq\left\|\mathcal{V}^{*}\right\| d(t, s)+\left\|\mathcal{V}^{*}-\mathcal{W}^{*}\right\| \\
& \leq d(t, s)+\|\mathcal{V}-\mathcal{W}\| .
\end{aligned}
$$


Since, $\left\|R_{|t|}-R_{|s|}\right\|=\left\|R_{t}-R_{s}\right\| \leq d(t, s)$ and $\left\|\mathcal{C}_{|t|}-\mathcal{C}_{|s|}\right\|=2 \tilde{d}(|t|,|s|) \leq$ $4 d(|t|,|s|)$ we have

$$
\begin{aligned}
\left\|\mathcal{C}_{|t|}-\mathcal{C}_{|s|}\right\| \leq 4 d(|t|,|s|) & =4 \sup \left\{\left\|R_{|t|}-R_{|s|}\right\|,\left\||t| R_{|t|}-|s| R_{|s|} \mid\right\|\right\} \\
& \leq 4 d(t, s)+4\|\mathcal{V}-\mathcal{W}\| .
\end{aligned}
$$

Corollary 3.3. Suppose $t, t_{n} \in R(E, F)$, for each $n \in \mathbb{N}$. Suppose $\mathcal{V}|t|$ is the polar decomposition of $t$, and for each $n, \mathcal{V}_{n}\left|t_{n}\right|$ is the polar decomposition of $t_{n}$.

(i) If $\left\|\mathcal{V}_{n}-\mathcal{V}\right\| \rightarrow 0,\left\|\mathcal{C}_{\left|t_{n}\right|}-\mathcal{C}_{|t|}\right\| \rightarrow 0$ and $\left\|\mathcal{C}_{\left|t_{n}^{*}\right|}-\mathcal{C}_{\left|t^{*}\right|}\right\| \rightarrow 0$ as $n \rightarrow+\infty$, then $t_{n} \rightarrow t$ in the gap topology.

(ii) If $\left\|\mathcal{V}_{n}-\mathcal{V}\right\| \rightarrow 0$ and $d\left(t_{n}, t\right) \rightarrow 0$ as $n \rightarrow+\infty$, then $\mathcal{C}_{\left|t_{n}\right|} \rightarrow \mathcal{C}_{|t|}$ in the norm topology.

Recall that a $\mathrm{C}^{*}$-algebra of compact operators is a $c_{0}$-direct sum of elementary $\mathrm{C}^{*}$-algebras $\mathcal{K}\left(H_{i}\right)$ of all compact operators acting on Hilbert spaces $H_{i}, i \in I$, i.e., $\mathcal{A}=c_{0}-\oplus_{i \in I} \mathcal{K}\left(H_{i}\right)$, cf. [1, Theorem 1.4.5]. If $\mathcal{A}$ is an arbitrary $\mathrm{C}^{*}$-algebra of compact operators then for every pair of Hilbert $\mathcal{A}$-modules $E, F$, every densely defined closed operator $t: \operatorname{Dom}(t) \subseteq E \rightarrow F$ is automatically regular and has polar decomposition, cf. [5-7]. These facts enable us to reformulate Theorem 3.2 and Corollary 3.3 in terms of densely defined closed operators on Hilbert $\mathrm{C}^{*}$-modules over $\mathrm{C}^{*}$-algebras of compact operators, or in terms of densely defined closed operators on Hilbert spaces.

Acknowledgements.

The authors would like to thank the referee for his/her useful comments. The author is also grateful to Professor M. Frank for his attention and some helpful comments.

\section{REFERENCES}

[1] W. Arveson, An Invitation to $\mathrm{C}^{*}$-algebras, Springer, New York, 1976.

[2] S. Baaj and P. Julg, Théorie bivariante de Kasparov et opérateurs non bornés dans les $C^{*}$-modules hilbertiens, C. R. Acad. Sc., Paris, Series I 296 (1983), 875-878.

[3] N. Castro-González, J. J. Koliha and V. Rakočević, Continuity and general perturbation of the Drazin inverse for closed linear operators, Abstr. Appl. Anal. 7 (2002), $335-347$.

[4] M. Frank, Geometrical aspects of Hilbert $C^{*}$-modules, Positivity 3 (1999), 215-243.

[5] M. Frank and K. Sharifi, Adjointability of densely defined closed operators and the Magajna-Schweizer theorem, J. Operator Theory 63 (2010), 271-282.

[6] M. Frank and K. Sharifi, Generalized inverses and polar decomposition of unbounded regular operators on Hilbert $C^{*}{ }^{-}$modules, J. Operator Theory 64 (2010), 377-386.

[7] B. Guljaš, Unbounded operators on Hilbert $C^{*}$-modules over $C^{*}$-algebras of compact operators, J. Operator Theory 59 (2008), no. 1, 179-192.

[8] T. Kato, Perturbation theory for linear operators, Springer Verlag, New York, 1984.

[9] J. J. Koliha and V. Rakočević, Continuity of the Drazin inverse II, Studia Math. 131 (1998), no. 2, 167-177. 
[10] E. C. Lance, Hilbert $C^{*}$-modules, LMS Lecture Note Series 210, Cambridge Univ. Press, 1995.

[11] B. Magajna, Hilbert $C^{*}$-modules in which all closed submodules are complemented, Proc. Amer. Math. Soc. 125(3) (1997), 849-852.

[12] K. Sharifi, The gap between unbounded regular operators, to appear in J. Operator Theory, available on arXiv:math.OA/0901.1891 v1 13 Jan 2009.

[13] K. Sharifi, Descriptions of partial isometries on Hilbert $C^{*}$-modules, Linear Algebra Appl. 431 (2009), 883-887.

[14] K. Sharifi, Topological approach to unbounded operators on Hilbert $C^{*}$-modules, to appear in Rocky Mountain J. Math.

[15] N. E. Wegge-Olsen, K-theory and $\mathrm{C}^{*}$-algebras: a Friendly Approach, Oxford University Press, Oxford, England, 1993.

[16] S. L. Woronowicz, Unbounded elements affiliated with $C^{*}$-algebras and noncompact quantum groups, Comm. Math. Phys. 136 (1991), 399-432.

K. Sharifi

Department of Mathematics

Shahrood University of Technology

P. O. Box 3619995161-316

Shahrood, Iran

E-mail: sharifi.kamran@gmail.com \& sharifi@shahroodut.ac.ir

Received: 20.7.2009.

Revised: 1.11 .2009 